\title{
Numerical Solution of First Order Ordinary Differential Equation by Using Runge-Kutta Method
}

\author{
Kedir Aliyi Koroche \\ Department of Mathematics, College of Natural and Computational Sciences, Ambo University, Ambo, Ethiopia
}

Email address:

kediraliyi39@gmail.com

\section{To cite this article:}

Kedir Aliyi Koroche. Numerical Solution of First Order Ordinary Differential Equation by Using Runge-Kutta Method. International Journal of Systems Science and Applied Mathematics. Vol. 6, No. 1, 2021, pp. 1-8. doi: 10.11648/j.ijssam.20210601.11

Received: December 20, 2020, 2020; Accepted: January 6, 2021; Published: March 10, 2021

\begin{abstract}
In this paper, the classical fourth-order Runge-Kutta methodis presented for solving the first-order ordinary differential equation. First, the given solution domain is discretizedby using a uniform discretization grid point. Next by applyingthe forward difference method, we discretized the given ordinary differential equation. And formulating a difference equation. Then using this difference equation, the given first-order ordinary differential equation is solved by using the classicalfourth-order Runge-Kutta method at each specified grid point. To validate the applicability of the proposed method, two model examples are considered and solved at each specific grid point on its solution domain. The stability and convergent analysis of the present method is worked by supportedthe theoretical and mathematical statementsand the accuracy of the solution is obtained. The accuracy of the present methodhas been shown in the sense ofmaximumabsolute error and the local behavior of the solution is captured exactly. Numerical and exact solutions have been presented in tables and graphs and the corresponding maximumabsolute errorisalso presented in tables and graphs. The present method approximates the exact solution very well and it is quite efficient and practically well suitedfor solving first-order ordinary differential equations. The numerical result presented in tables and graphsindicates that the approximate solution is in good agreement with the exact solution. Hence the proposed method is accruable to solve ordinary differential equations.
\end{abstract}

Keywords: Ordinary Differential Equation, Runge-Kutta Method, Initial Value Problem, Boundary Value Problem, Stability and Convergent Analysis, Maximum Absolute Error

\section{Introduction}

Numerical analysis is a subject that involves computational methods for studying and solving mathematical problems. It is a branch of mathematics and computer science that creates, analyzes, and implements algorithms for solving mathematical problems numerically.

Numerical methods usually emphasize the implementation of numerical algorithms. The aim of these numerical methods is, therefore, to provide systematic techniques for solving mathematical problems numerically. Numerical methods are well suited for solving mathematical problems by using modern digital computers, which are very fast and efficient in performing arithmetic operations. The process of solving problems using high precision digital computers generally involves starting from initial data; the concerned appropriate algorithms are then executed to yield the required results [1].

The ultimate aim of the field of numerical analysis is to provide convenient methods for obtaining the solutions to mathematical problems and for extracting useful information from available solutions that are not expressed intractable forms. Such problems may be formulated in terms of algebraic equations, transcendental equations, ordinary differential equations, and partial differential equations $[2,3]$.

The ordinary differential equation is a mathematical equation that is significant in physical phenomena that occur in nature. The majority of ordinary differential equations difficult to approximate by analytical methods. Its, therefore, important to be able to obtain an accurate solution numerically [1].

Numerical methods for the solution of ordinary differential equations may be put in two categories-numerical integration (e.g., predictor-corrector) methods and Runge-Kutta methods. The advantages of the latter are that they are self-starting and easy to program for digital computers but neither of these reasons is very compelling when library subroutines can be written to handle 
systems of ordinary differential equations. Thus, the greater accuracy and the error-estimating ability of predictor-corrector methods make them desirable for systems of any complexity. However, when predictor-corrector methods are used, RungeKutta methods still find application in starting the computation and in changing the interval of integration [11]. One of this method Runge-Kutta 4th order method is a numerical technique used to solve ordinary differential equations [9]. Runge-Kutta methods are considered in the context of using them for starting and for changing the interval, matters such as stability and minimization of roundoff errors are not significant [11].

Traditionally, methods such as the Taylor series method, Euler method, RungeKutta second \& third order methods, and finite difference method, have been used to approximate first-order ordinary differential equation. Despite their great success in the past decades in mathematics problems, these methods require time to consume to solve ordinary differential equations. The costs and difficulties in creating quality solutions, however, constitute one of the major bottlenecks in these methods. However, the complications of these methods include a slow rate of convergence, instability, low accuracy. To this end, this Project aims to develop a fourth-order Runge-Kuttamethod that is capable of producing an accurate solution of first-order ordinary differential equations on a specific given domain.

In this work, we propose a numerical method for solving the first-order ordinary differential equation and the convergence has been shown in the sense of absolute errors that the local behavior of the solution is captured exactly.

\section{Objectives of the Study}

a) General Objective

The general objective of this study is to develop a classical fourth-order Runge-Kutta method for solving the first-order ordinary differential equation

b) Specific Objectives

The specific objectives of the study are:

1. To formulate the numerical method for solving firstorder ordinary differential equation;

2. To test the extent to which the proposed method approximates the exact solution;

3. To describe the advantage of the present method over another method.

\section{Significance of the Study}

The outcome of this study provides some background information for other researcherswho work on this and the related area of study It also introduces the application of numerical methods in different fields of study.

\section{Delimitation of the Study}

This study is delimited to the fourth-order Runge-Kutta method for solving first-order ordinary differential equation given in the form of:

$$
y^{\prime}=f(x), y\left(x_{0}\right), a \leq x \leq b
$$

\section{Review of Related Literature}

Many problems in science and engineering can be formulated in terms of differential equations. A differential equation is an anequation involving a relation between an unknown function and one or more of its derivatives [17].

Most fundamental laws of Science are based on models that explain variations in physical properties and states of systems described by differential equations [5]. An equation that consists of derivatives is called a differential equation. Differential equations have applications in all areas of science and engineering. The mathematical formulation of most of the physical and engineering problems leads to differential equations. So, engineers and scientists need to know how to set up differential equations and solve them [11]. This differential equation is categorized into two parts. These two parts are the Ordinary differential equation and the Partial differential equation $[1,2,10]$. An ordinary differential equation is a differential equation that involves derivatives of one or more dependant variables concerning at most one independent variable [1, 2, 17]. A partial differential equation is a differential equation that involves the derivative of one or more dependant variables concerning two or more than two variables [2]. It is now well established that numerical schemes for solving ordinary differential equations (ODEs) may exhibit perioddoubling and chaotic behavior when used with time steps above their linearised stability limit [16]. An Ordinary differential equation is categorized into the Initial value problem and boundary value problem $[1,2,17]$.

\subsection{Initial Value Problem}

Initial value problems for ordinary differential equations (ODEs) occur in almost all the sciences, notably in mechanics (including celestial mechanics), where the motion of particles (resp., planets) is governed by Newton's second law - a system of second-order differential equations. It is no wonder, therefore, that astronomers such as Adams, Moulton, and Conwell were instrumental in developing numerical techniques for their integration. Still, when separated in polar coordinates, it reduces to an ordinary second-order linear differential equation. Such equations are at the heart of the theory of special functions of mathematical physics [4].

Within mathematics, ordinary differential equations play an important role in the calculus of variations, where optimal trajectories must satisfy the Euler equations, Orin optimal control problems, where they satisfy the Pontryaginmaximum principle.

In both cases, one is led to boundary value problems for ordinary differential equations [4]. Initial Value Problem is types of ode that given Information at a single value of the independent variable, typically at the beginning of the interval [5]. This initial value problem is given in the form of $y^{\prime}=\frac{d y}{d x}=f(x), y\left(x_{0}\right)=y_{0}, a \leq x \leq b$, for $\mathrm{x}$ is an 
independent variable and $x_{0}=a$. Different numerical methods used to solve these types of problems, like the Euler method, the Taylor series method, and finite difference method. But this numerical method is a low convergence rate and consumes time. So that we develop and use the convergent numerical method which is called the classical fourth-order Runge-Kutta method.

\subsection{Boundary Value Problem (BV)}

Many problems in applied mathematics require solutions of differential equations specified by conditions at more than one point of the independent variable. These are called boundary value problems; they are considerably more difficult to deal with than initial value problems, largely because of their global nature. Unlike (local) existence and uniqueness theorems known for initial value problems and there are no comparably general theorems for boundary value problems. Neither existence nor uniqueness is, in general, guaranteed [4].

The finite difference method is used to solve ordinary differential equations that have conditions imposed on the boundary rather than at the initial point. These problems are called boundary-value problems. Many academics refer to boundary value problems as position-dependent and initial value problems as time-dependent [8].

We concentrate here on two-point boundary value problems, in which the systemof differential equations $y^{\prime}=$ $\frac{d y}{d x}=f(x), y\left(x_{0}\right)=y_{0}, y\left(x_{n}\right)=y_{n}, a \leq x \leq b$, for $x$ is an independent variable $x_{0}=a, x_{n}=b$ and both $y_{0}$ and $y_{n}$ are boundary conditions given at boundary points of specific domains respectively $a$ and $b$. This problem has different applications in the real-life world. In this project, we discuss the solution of initial value problem solving by using the Runge-Kutta method [4].

\subsection{Runge Kutta Methods}

Numerical methods for the solution of ordinary differential equations may be put in two categories-numerical integration (e.g., predictor-corrector) methods and Runge-Kutta methods. The advantages of the latter are that they are selfstarting and easy to program for digital computers but neither of these reasons is very compelling when library subroutines can be written to handle systems of ordinary differential equations. Thus, the greater accuracy and the error-estimating ability of predictor-corrector methods make them desirable for systems of any complexity. However, when predictorcorrector methods are used, Runge-Kutta methods still find application in starting the computation and in changing the interval of integration [11]. Runge-Kutta methods are standard in the fields of numerical ODEs [12].

One can distinguish between analytic approximation methods and discrete-variable methods. In the former, one tries to find approximations to the exact solution $y_{a p}(x) \approx y(x)$, valid for all $x \in(a, b)$ where $y_{a p}$ approximate value and $y(x)$ exact values of the given problem. These usually take the form of a truncated series expansion, either in powers of $x$, in Chebyshev polynomials, or some other system of basic functions. So to approximate this solution by using a single step and multistep methods. Single-step methods for IV problems are Euler, Heun, and Midpoint/Improved Polygon and general Runge-Kutta methods. Multi-step methods for IV problems are Newton-cotes Adams methods [3, 4]. To solve the initial value problem is given in the form of $y^{\prime}=\frac{d y}{d x}=$ $f(x), y\left(\mathrm{x}_{0}\right)=\mathrm{y}_{0}, \mathrm{a} \leq \mathrm{x} \leq \mathrm{b}$, key to the various one-step methods is how the slope is obtained. This slope represents a weighted average of the slope over the entire interval and may not be the tangent at $\left(x_{i}, y_{i}\right)[5]$. Hence in our work, we use Runge-Kutta methods. Employing single-step

$$
y_{i+1}=y_{j}+h \varphi\left(x_{i}, y_{j}, h\right)
$$

Increment function or slope, $\varphi\left(x_{i}, y_{j}, h\right)$ is a weighted average: $\varphi=a_{1} k_{1 i}+a_{2} k_{2 j}+\cdots+a_{j} k_{j i}+\cdots+a_{n} k_{n i}\left(n^{t h}\right.$ order methods) where: $a_{j}=$ weighting factors (that sum to unity)

$$
\begin{gathered}
k_{1 i}=h f\left(x_{i}, y_{j}\right) \\
k_{2 i}=h f\left(x_{i}+p_{1} h+, y_{j+} q_{11} k_{1 i}\right), \\
k_{2 i}=h f\left(x_{i}+p_{2} h+, y_{j+} q_{21} k_{1 i}+q_{22} k_{2 i}\right) \\
\ldots \ldots \ldots \\
k_{2 i}=h f\left(x_{i}+p_{n-1} h+, y_{j+} q_{n-11} k_{1 i}+q_{n-12} k_{2 i}+\cdots\right. \\
\left.+q_{n-1 n-1} k_{n-1 i}\right)
\end{gathered}
$$

Now from this general $n$ stage Runge-Kutta Methods, the classical fourth-order Runge-Kutta method employing singlestep given as:

$$
y_{i+1}=y_{i}+1 / 6\left[k_{1}+2 k_{2}+2 k_{3}+k_{4}\right]
$$

where $k_{1}=h f\left(x_{i}, y_{j}\right), k_{2}=h f\left(x_{i}+\frac{1}{2} h+, y_{j+} \frac{1}{2} k_{1}\right), k_{3}=$ $h f\left(x_{i}+\frac{1}{2} h+, y_{j+} \frac{1}{2} k_{2}\right), k_{2}=f\left(x_{i}+h+, y_{j+} k_{3}\right)$. Global truncation error, $O\left(h^{4}\right)$. For our discussion, we shall consider This explicit Runge-Kutta method only. However, Runge-Kutta methods must compare with the Taylor series method when they are expanded about the point $x=x i$.

\section{Study Area and Period}

This study is conducted at the Ambo university's department of mathematics in 2020 G. C. conceptually; the study focused on the classical fourth-order Runge-Kutta method for solving a first-order ordinary differential equation.

\section{i) Study Design}

This study employed a documentary review and numerical experimental design.

\section{ii) Source of Information}

This study utilized information from books, Google/ internet, and the experimental results obtained through coding.

\section{iii) Mathematical Procedures}

To achieve the stated objectives, the study followed the following steps: 1) Defining the problem or formulating of the problem; 2) Discrediting the solution domain; 3) Solving firstorder ordinary differential equation by using the method4) 
Validating the method via some numerical example.

\section{Description of Numerical Experiment}

In this section, we use the fourth-order RungeKutta method for finding the approximate solutions of the initial value problem (IVP) of the first-order ordinary differential equation has the form

$$
y^{\prime}=f(x, y), x \in\left(x_{0}, x_{n}\right), y\left(x_{0}\right)=y_{0}
$$

where $y^{\prime}=\frac{d y}{d x}, f(x, y)$ is given smooth faction and $y(x)$ is the solution of Equation (1). Numerical methods employ Equation (1) to obtain approximations to the values of the solution corresponding to various selected values of $x_{n}=$ $x_{0}+n h,=1,2,3, \ldots, N-1$. The parameter $h$ is called the step size. The numerical solutions of (1) are given by a set of points $\left\{\left(x_{n}, y\left(x_{n}\right)\right), n=0,1,2, \ldots \ldots\right\}$ and each point $\left(x_{n}, y_{n}\right)$ is an approximation to the corresponding point $\left(x_{n}, y\left(x_{n}\right)\right)$ on the solution curve [6].

Stability and Convergent Analysis.

In contrast to the form (1), we shall consider equations of the form

$$
y^{\prime}=A y+\psi(x, y)
$$

It is assumed that $\psi$ satisfies a Lipchitz condition with constant $C$ such that:

$$
\|\psi(x, u)-\psi(x, v)\| \leq C\|u-v\|
$$

This form includes the ones traditionally considered. Associated with Eq. (1) is the "model problem,"

$$
y^{\prime}=A y
$$

Because the model problem is comparatively easy to analyze in detail, we shall view Eq. (1) as arising from the addition of a (possibly) nonlinear term to the equation Eq. (3). We shall continually ask what happens for the model problem and then ask to what degree the general problem shares this behavior.

For the sake of simplicity and definiteness, it is assumed that the matrix ' $A$ ' can be diagonalized by a similarity transformation

$$
P A P^{-1}=\Lambda=\operatorname{diag}\left(\lambda_{j}\right)
$$

It is then easy to analyze the stability of the model problem. Assumed that the vector norm implicit in Eq. (2) is the Euclidean norm. The associated subordinate matrix norm will be used. Our analysis is based on another vector norm, the P-norm, defined by

$$
\|v\|_{m} \leq\|P v\|
$$

where $\mathrm{P}$ is the matrixes given in Eq. (5). Its subordinate matrix norm is

$$
\|G\|_{p} \leq\left\|P v P^{-1}\right\|
$$

Inequalities in one norm are connected to inequalities in the other by the condition number of 'P', $k(A)=\|P\|\left\|P^{-1}\right\|$

$\|v\| \leq\|u\|$ Implies $\|v\| \leq k(A)\|u\|$ and $\|v\|_{p} \leq\|u\|_{p}$ implies $\|v\|_{p} \leq k(A)\|u\|_{p}$.

In particular, Eq. (3) implies

$$
\|\psi(x, u)-\psi(x, v)\|_{p} \leq C k(A)\|u-v\|_{p}
$$

With these preliminary observations it is now easy weobtain a stability result for the model problem.

Lemma 1: Let $\breve{y}(x), y(x)$ be two solutions toEq. (4). If $A$ satisfies Eq. (5), then

$$
\left\|\check{y}\left(x_{n}\right)-y\left(x_{n}\right)\right\|_{p} \leq\left\|\check{y}\left(x_{n}\right)-y\left(x_{n}\right)\right\|_{p} e^{\eta\left(x-x_{n}\right)}
$$

Where

$$
\eta=\operatorname{rel}\left(\lambda_{j}\right)
$$

Proof: Any solutiony $(x)$ of Eq. (4) can be written as

$$
y(x)=y\left(x_{n}\right) e^{A\left(x-x_{n}\right)}
$$

from which it is obvious that

$$
\left\|\check{y}\left(x_{n}\right)-y\left(x_{n}\right)\right\|_{p} \leq\left\|e^{A\left(x-x_{n}\right)}\right\|\left\|\left(\check{y}\left(x_{n}\right)-y\left(x_{n}\right)\right)\right\|_{p}
$$

At this point the roles played by the assumption Eq. (5) and the P-norm are seen:

$$
\begin{aligned}
& || e^{A\left(x-x_{n}\right)}||_{P} \leq\left\|p e^{A\left(x-x_{n}\right)} p^{-1}\right\| \\
& =\left\|\operatorname{diag}\left(e^{\lambda_{j}\left(x-x_{n}\right)}\right)\right\|=e^{\eta\left(x-x_{n}\right)}
\end{aligned}
$$

The standard theory applied to the model problem (4) leads to (6) with

$$
C=\|A\|_{P}=\left\|\operatorname{diag}\left(\lambda_{j}\right)\right\|=\max \left(\lambda_{j}\right)=\rho(A)
$$

Where $\rho(A)$ is the spectra radius of coefficient matrix $A$.

The result Eq. (7) is advantageous when $\eta \leq \rho(A)$. A difference between the two approaches is made clear on considering the two scalar problems y' $=$ Ay and y ' $=-$ Ay. The classical theory does not distinguish these problems and Lemma 1 does. According to Eq. (7), the integral curves of the model problem do not spread apart at all if $\eta<0$ and come together if $\eta<0$. More generally, an equation Eq. (2) is said to be contractive in the P-norm if

$$
\|\check{y}(x)-y(x)\|_{p} \leq\left\|\left(\check{y}\left(x_{n}\right)-y\left(x_{n}\right)\right)\right\|_{p}
$$

for all pairs of distinct solutions $\breve{y}(x)$ and $y(x$.)

Lemma 2: If A satisfies Eq. (5), the model problem Eq. (4) is contractive in the p-norm if, and only if, $\eta<0$.

Proof. One direction is an immediate consequence of (7). Suppose now that $\breve{y}(x)-y(x)=v_{i}$ is an eigenvector of Acorresponding to the eigenvalues $\lambda_{j}$. Then

$$
\begin{gathered}
\left.\|\check{y}(x)-y(x)\|_{p}=|| v_{i} e^{\left(\lambda_{j}\left(x-x_{n}\right)\right.}\right)||= \\
\left|e^{\left(\lambda_{j}\left(x-x_{n}\right)\right.}\right|\left\|\left(\check{y}\left(x_{n}\right)-y\left(x_{n}\right)\right)\right\|_{p}
\end{gathered}
$$

Clearly, these two solutions do not approach one another 
unless $\operatorname{real}\left(\lambda_{j}\right)<0$ and we conclude that necessarily $\eta<0$. Now let us derive a stability result like Eq. (7) for the whole class Eq. (2). The classical variation of constants formula provides a generalization of Eq. (5) which exposes the roles of $\mathrm{A}$ and $\psi$ :

$$
y(x)=y\left(x_{n}\right) e^{A\left(x-x_{n}\right)}+\int_{x_{n}}^{x} e^{(A(x-t)} \psi(t, y(t)) d t
$$

Theorem 1: Let $\breve{y}(x), y(x)$ be two solutions of Eq. (1). If $A$ satisfies Eq. (5) and $\psi$ satisfies Eq. (3), then

$$
\|\check{y}(x)-y(x)\|_{p} \leq\left\|\check{y}\left(x_{n}\right)-y\left(x_{n}\right)\right\|_{p} e^{\left(x-x_{n}\right)(\eta+C k(P))}
$$

Proof. Let

$$
\varphi(x)=\|\check{y}(x)-y(x)\|_{p}
$$

Then by the Lipchitz and the representation Eq. (9) lead easily to

$$
\varphi(x) \leq \varphi\left(x_{n}\right)\left\|e^{A\left(x-x_{n}\right)}\right\|_{p}+\int_{x_{n}}^{x}\left\|e^{A(x-t)}\right\|_{P} C k(P) \varphi(t) d t
$$

On bounding the norms of the exponential matrices as in the proof of Lemma 1 and on introducing

$$
\delta(x)=\varphi(x) e^{-\eta x}
$$

it is found that

$$
\delta(\boldsymbol{x}) \leq \boldsymbol{\delta}\left(\boldsymbol{x}_{\boldsymbol{n}}\right)+\int_{x_{n}}^{x} C k(P) \delta(t) d t
$$

Gronwall's inequality then states that

$$
\delta(\boldsymbol{x}) \leq \boldsymbol{\delta}\left(\boldsymbol{x}_{\boldsymbol{n}}\right) e^{l k(p)\left(x-x_{n}\right)} .
$$

which is equivalent to the result desired.

Corollary 1: With the assumptions of Theorem 1, the problem (2) is contractive in the P-norm if

$$
\eta+C k(p)<0
$$

Theorem 1 includes the classical result when $\mathrm{A}=0$. It is more informative and is obtained at the price of analysis only a little more complicated. The most interesting situation is when A has Eigen-values $\lambda_{j}$ with $\left|\lambda_{j}\right|$ large and $\operatorname{Re}\left(\lambda_{j}\right)<$ 0 because we obtain than much more realistic stability bound. We have an attractive formulation of contractility: If the model problem is contractive in the P-norm, so is the general problem for all sufficiently small C. In any case, we see that for "small" C, the stability bound is much the same as that for the model problem.

Theorem 2: In our case, the classical fourth-orderRungeKutta method is applied to solve ODE in Eq. (1) so it has the representation of the form:

$$
u_{i}=K_{i}(h A) u_{0}+\sum_{j-0}^{i-1} K_{i j}(h A) \psi\left(x_{j}, u_{j}\right), j=1,2, \ldots 4
$$

$$
y_{n+1}=u_{5}=K(h A) u_{0}+h \sum_{j=0}^{4} K_{5 j}(h A) \psi\left(x_{j}, u_{j}\right)
$$

where the polynomials $K_{i}(z), K(z)$. and $K_{i j}(z)$ are defined by

$$
\begin{gathered}
K_{i}(z)=1+\mathrm{z} \sum_{\mathrm{j}=0}^{\mathrm{i}-1} \beta_{\mathrm{ij}} \mathrm{K}_{\mathrm{j}}(\mathrm{z}), \mathrm{i}=1,2, . .4 . \\
K(z)=1+\mathrm{z} \sum_{\mathrm{j}=0}^{4} \alpha_{\mathrm{i}} \mathrm{K}_{\mathrm{i}}(\mathrm{z}) \\
K_{i j}(z)=\beta_{\mathrm{ij}}+\mathrm{z} \sum_{\mathrm{l}=\mathrm{j}+1}^{\mathrm{i}-1} \beta_{\mathrm{i} 1} \mathrm{~K}_{1 \mathrm{j}}(\mathrm{z}), 0 \leq \mathrm{j}<i \leq 4 \\
K_{5 j}(z)=\alpha_{\mathrm{i}}+\mathrm{z} \sum_{\mathrm{l}=\mathrm{j}+1}^{4} \alpha_{\mathrm{i} 1} \mathrm{~K}_{1 \mathrm{j}}(\mathrm{z}), \mathrm{j}=0,1,2,3,4 .
\end{gathered}
$$

Lemma 3: Let $\breve{y}_{n+1}, y_{n+1}$ be the results of applying the Runge-Kuttamethod to the model problem Eq. (2) starting with the approximations $\breve{y}_{n}, y_{n}$, respectively. Suppose that J satisfies (5) and that $\eta<0$. Then

$$
\left\|\check{y}_{n+1}-y_{n+1}\right\|_{p} \leq\left\|\check{y}_{n}-y_{n}\right\|_{p}
$$

for all $\check{y}_{n}, y_{n}$ if, and only if, $h \lambda \in \mathrm{B}$ for all eigenvalues of $\mathrm{A}$

Proof: LetK be stability polynomial and B be a (compact) region in the complex plane defined by $B=\{z: \operatorname{Real}(z) \leq$ $0\}$ and $|K(z)| \leq 1$. Then from Eq. (7) we have

$$
\begin{gathered}
\left\|\check{y}_{n+1}-y_{n+1}\right\|_{p}=\left\|\mathrm{K}(\mathrm{hA})\left(\check{y}_{n}-y_{n}\right)\right\|_{p} \\
\leq\|\mathrm{K}(\mathrm{hA})\|_{p}\left\|\check{y}_{n}-y_{n}\right\|_{p}
\end{gathered}
$$

Now if each $h \lambda_{j} \in B$, this proves sufficient par. If $\check{y}_{n}-y_{n}$ is an eigenvector $v_{j}$ of $A$ corresponding to the eigenvalue $\lambda_{j}$ then

$$
\begin{gathered}
\left\|\breve{y}_{n+1}-y_{n+1}\right\|_{\boldsymbol{p}}=\left\|\mathrm{k}\left(\mathrm{h} \lambda_{j}\right)\left(\breve{y}_{n}-y_{n}\right)\right\|_{\boldsymbol{p}} \\
=\left\|\mathrm{k}\left(\mathrm{h} \lambda_{j}\right)\right\|_{\boldsymbol{p}}\left\|\check{y}_{n}-y_{n}\right\|_{\boldsymbol{p}}
\end{gathered}
$$

From which it is seen that $\left|\mathrm{k}\left(\mathrm{h} \lambda_{j}\right)\right| \leq 1$ is necessary. Hence for $\eta<0$ implies $h \lambda_{j} \in B$, the general solution of Eq. (1) is stable.

Theorem 3: Let $\left\{\breve{u}_{i}\right\}, \breve{y}_{n+1}$ and $\{u\}_{i}, y_{n+1}$ are the solution of Eq. (1) obtained by classical range Kutta method starting with the approximations $\mathrm{g} \breve{y}_{n}$ and, $y_{n}$, respectively. Suppose that A satisfies Eq. (5) and $\psi$ satisfies Eq. (3). Suppose $\operatorname{Re}\left(\lambda_{j}\right) \leq 0$ for all eigenvalue $\lambda_{j}$ of A that is $\operatorname{Re}\left(\lambda_{j}\right)=0$, for only $\lambda_{j}=0$. Suppose further that $h \lambda_{j} \in B$ for all $\lambda_{j}$. Then

$$
\begin{gathered}
\left\|\check{u}_{i}-u_{i}\right\|_{P} \leq\left\|K_{i}^{*}+h H_{i}\right\|_{P} i=1,2,3, \ldots m \\
\left\|\check{y}_{n+1}-y_{n+1}\right\|_{P} \leq\left(K_{i}^{*}+h L .\right)\left\|\check{y}_{n}-y_{n}\right\|_{P} \\
\left\|\check{y}_{n+1}-y_{n+1}\right\|_{P} \leq(1+h C k(P) \Upsilon(h) .)\left\|\check{y}_{n}-y_{n}\right\|_{P}
\end{gathered}
$$

Where $K_{i j}^{*}=\max _{z \in B}\left|K_{i j}(z)\right|, 0 \leq j<i \leq 5$

$$
K_{i}^{*}=\max _{z \in B}\left|K_{i}(z)\right|, 0 \leq i \leq 4
$$




$$
\begin{array}{cc}
K_{.}^{*}=\max _{z \in B}\left|K_{i}(z)\right|=1 & \Upsilon(h)=C k(p) \sum_{j=0}^{4} K_{5 j}^{*}\left(K_{j}^{*}+h L_{j}\right) \\
H_{i}=C k(p) \sum_{j=0}^{i-1} K_{i j}^{*}\left(K_{i}^{*}+h L_{j}\right) 0 \leq j \leq 4 & \text { Where } K_{i}(z), K(z) \text {. and } K_{i j}(z) \text { are defined in Theorem } 2 . \\
\check{u}_{1}-u_{1}=K_{1}(h A)\left(\check{u}_{0}-u_{0}\right)+h K_{1,0}(h A)\left[\psi\left(x_{0}, \breve{u}_{0}\right)-\psi\left(x_{0}, u_{0}\right)\right]
\end{array}
$$

Using the Lipchitz condition Eq. (6) satisfied by $\psi$ one finds

$$
\left\|\check{u}_{1}-u_{1}\right\|_{p} \leq\left(\left\|K_{1}(h A)+h C k(P)\right\|_{p} K_{1,0}(h A)\right)\left\|\check{u}_{0}-u_{0}\right\|_{p}
$$

Then

$$
\left\|K_{1}(h A)\right\|_{p}=\left\|\operatorname{diag}\left\{K_{1}(h A)\right\}\right\|=\max _{l}\left|K_{1}\left(h \lambda_{j}\right)\right| \leq K_{i}^{*}
$$

and similarly,

$$
\left\|K_{1,0}(h A)\right\|_{p} \leq K_{l 0}^{*}
$$

Because $K_{0} \equiv 1, H_{0}=0$, the conclusion of the theorem is seen to hold for $i=1$. Suppose now that the statement holds through index $i-1$. Then

$$
\breve{u}_{i}-u_{i}=K_{i}(h A)\left(\check{u}_{0}-u_{0}\right)+h \sum_{j-0}^{i-1} K_{i j}(h A)\left[\psi\left(x_{j}, u_{j}\right)-\psi\left(x_{j}, u_{j}\right)\right]
$$

Hence taking matrixes norm on both sides this equation, we obtain:

$$
\begin{gathered}
\left\|\check{u}_{i}-u_{i}\right\|_{p} \leq\left\|K_{i}(h A)\right\|_{p}\left\|\left(\check{u}_{0}-u_{0}\right)\right\|_{p}+h \sum_{j-0}^{i-1}\left\|K_{i j}(h A)\right\|_{p} C k(P)\left\|\check{u}_{j}-u_{j}\right\|_{p} \\
\leq\left(K_{i}^{*}+h C k(P) \sum_{j-0}^{i-1} K_{i j}^{*}\left(K_{i}^{*}+h L_{j}\right)\left\|\check{u}_{0}-u_{0}\right\|_{p}\right. \\
\leq\left(K_{i}^{*}+h L_{j}\left\|\check{u}_{0}-u_{0}\right\|_{p}\right.
\end{gathered}
$$

The result for $y_{n+1}$ is verified in the same way. Hence from this, the global error in our scheme of classical RungeKutta method is bounded, so the scheme is stable. Hence the proposed method is convergent. Farther to more understanding stability and convergent Analysisof RungeKutta method of stage s you con seereference [18].

\section{Investigating the Accuracy of the Method}

In this section, we investigate the accuracy of the present method. There are two types of errors in the numerical solution of ordinary differential equations. Round-off errors and Truncation errors occur when ordinary differential equations are solved numerically. Rounding errors originate from the fact that computers can only represent numbers using a fixed and limited number of significant figures. Thus, such numbers or cannot be represented exactly in computer memory. The discrepancy introduced by this limitation is called a Round-off error. Truncation errors in numerical analysis arise when approximations are used to estimate some quantity. The accuracy of the solution will depend on how small we make the step size, $h$. To show the accuracy of the present method, the maximum absolute error $e_{r}$ is used to measure the accuracy of the method. The maximum absolute error [7] is calculated by $e_{r}=\max _{1 \leq n \leq N}\left(\left|y\left(x_{n}\right)-y_{n}\right|\right)$ where $N$ is the maximum number of step, $y\left(x_{n}\right)$ is exact and $y_{n}$ approximation solution of IVP in Eq. (1) at the grid point $x_{n}$.

\section{Numerical Experiments}

Numerical results and errors are computed and the outcomes are represented tabularly and graphically.

Example 1: solve IVP given by $\frac{d y}{d x}=\frac{x}{y}, y(0)=1$, and its exact solution is $y=\sqrt{x^{2}+1}$ for $0 \leq x \leq 1$.

Example 2: Consider the initial value problem $\frac{d y}{d x}=$ $x(1+y), y(0)=1$. Compute $y(0.8)$ with $\mathrm{h}=0.1$. The exact solution is $y(x)=-1+2 e^{x^{2} / 2}$. 
Table 1. Computations numerical and exact solution for the first order initial value problem given in example 1 and listing their corresponding maximum absolute errors.

\begin{tabular}{llll}
\hline $\mathbf{X} \_$(grid point) & y_(Approximate solution) & y_(Exact solution) & er_(absolute error) \\
\hline 0 & 1.000000000000000 & 1.000000000000000 & 0 \\
0.1 & 1.004987572425429 & 1.004987562112089 & $1.0313 \mathrm{e}-08$ \\
0.2 & 1.019803941472437 & 1.019803902718557 & $3.8754 \mathrm{e}-08$ \\
0.3 & 1.044030729213235 & 1.044030650891055 & $7.8322 \mathrm{e}-08$ \\
0.4 & 1.077033081960091 & 1.077032961426901 & $1.2053 \mathrm{e}-07$ \\
0.5 & 1.118034147228578 & 1.118033988749895 & $1.5848 \mathrm{e}-07$ \\
0.6 & 1.166190567243875 & 1.166190378969060 & $1.8827 \mathrm{e}-07$ \\
0.7 & 1.220655770449989 & 1.220655561573370 & $2.0888 \mathrm{e}-07$ \\
0.8 & 1.280625068576675 & 1.280624847486570 & $2.2109 \mathrm{e}-07$ \\
0.9 & 1.345362631261068 & 1.345362404707371 & $2.2655 \mathrm{e}-07$ \\
1 & 1.414213789413370 & 1.414213562373095 & $2.2704 \mathrm{e}-07$ \\
\hline
\end{tabular}

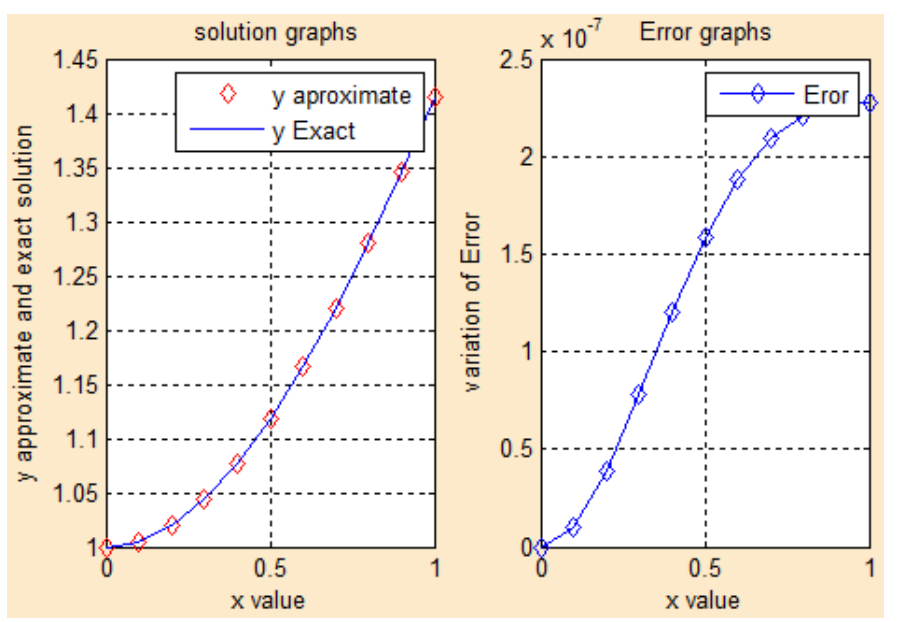

Figure 1. Graphs of the numerical and exact solution of example one and their absolute error expiration.

Table 2. Computations numerical and exact solution for the first order initial value problem given inexample 2 and listing their corresponding maximum absolute errors.

\begin{tabular}{llll}
\hline $\mathbf{X} \_$(grid point) & y_(Approximate solution) & y_(Exact solution) & er_(absolute error) \\
\hline 0 & 1.000000000000000 & 1.000000000000000 & 0 \\
0.1 & 1.010025041666667 & 1.010025041718802 & $5.2135 \mathrm{e}-11$ \\
0.2 & 1.040402679516737 & 1.040402680053512 & $5.3677 \mathrm{e}-10$ \\
0.3 & 1.092055717771941 & 1.092055719817434 & $2.0455 \mathrm{e}-09$ \\
0.4 & 1.166574129640990 & 1.166574135349917 & $5.7089 \mathrm{e}-09$ \\
0.5 & 1.266296892235075 & 1.266296906133653 & $1.3899 \mathrm{e}-08$ \\
0.6 & 1.394434694825263 & 1.394434726243620 & $3.1418 \mathrm{e}-08$ \\
0.7 & 1.555242558938220 & 1.555242626409774 & $6.7472 \mathrm{e}-08$ \\
0.8 & 1.754255389803884 & 1.754255528671914 & $1.3887 \mathrm{e}-07$ \\
\hline
\end{tabular}

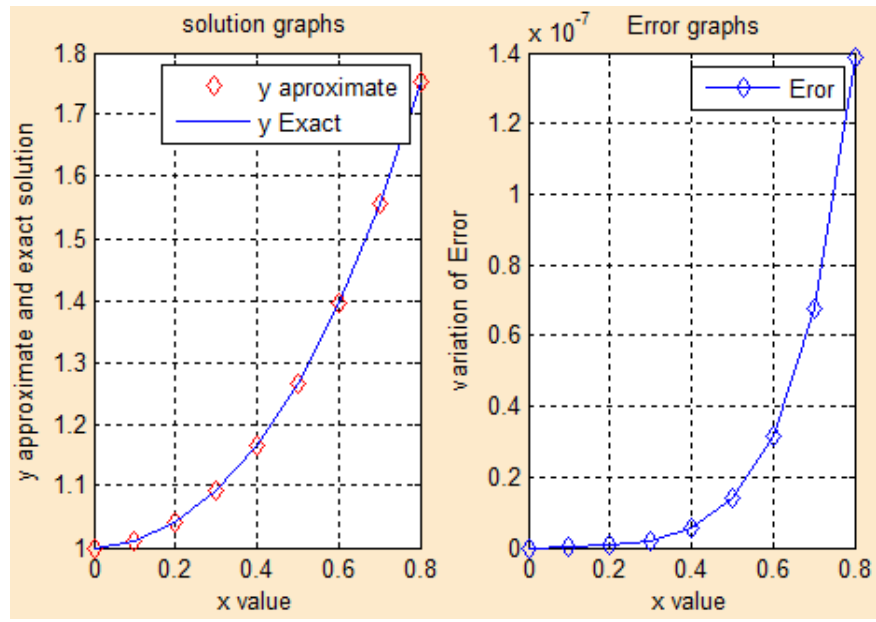

Figure 2. Graphs of the numerical and exact solution of example two and their absolute error expiration. 


\section{Discussion}

In this project paper, we Appling the classical fourth-order Runge-Kutta method for solving a first-order ordinary differential equations. To demonstrate the competence of the method, we applied it to two model examples. Numerical results obtained by the present method have been associated with their exact solution and the results are summarized in Tables and graphs. Ascan be seen from the numerical results predicted in the tables and graphs above, the present method is approximate the exact solution very well. To further verify the applicability of the planned method, graphs were plotted for the above examples for exact solutions versus the numerical solutions with correspondence absolute errors. As figure 1 indicates good agreement of approximation of exact solution for example 1. Figure 2 indicate the numerical solution has a good agreement of the results with the exact solution for example 2. Further, the numerical solutions presented in this Project paper validate the improvement of the numerical approximation for the solution of the firstorder ordinary differential equation.

\section{Conclusion}

In this project paper, we have used classical fourth-order Runge- Kutta methods for solving initial value problems (IVP). To achieve the desired accuracy of the numerical solution it is necessary to take is smallstepsize. It means the accuracy increase the step length decrease. Therefore, it is an important task to make a proper balance between the accuracy andefficiency of the solutions. Further, the numerical solutions presented in this Project paper validate the improvement of the numerical approximation for the solution of the first-order ordinary differential equation.

\section{Acknowledgements}

First of all, I would like to express my thanks to God, forgiving me for life and the time to accomplish my tasks. In the name of God, The Most Greatest and Most Merciful, there is no power except by the power of God and I humbly return my acknowledgment that all knowledge belongs to god. I thanksGod for granting me this opportunity to broaden my knowledge in this field. Nothing is possible unless $\mathrm{He}$ made it possible.

Secondly, greatly appreciate the help of my ShamoBusho and my father AliyiKorochee, without the help of them I would not be here today.

Last but not least, thanks a lot to all my beloved friends; your kindness and helps will be a great memory for me.

\section{References}

[1] Ray, S. (2018). Numerical analysis with algorithms and programming. CRC Press.
[2] Francis B. Hildebrand (1987). Introduction to numerical analysis, Second edition

[3] Sastry, S. S. (2006). Introductory method of numerical analysis, Fourth-edition.

[4] Walter Gautschi (2012) Numerical Analysis Second Edition

[5] Butcher, J. C., \& Goodwin, N. (2008). Numerical methods for ordinary differential equations (Vol. 2). New York: Wiley.

[6] Md. Amirul Islam. "A Comparative Study on numerical Solutions of Initial Value Problems (IVP) for Ordinary Differential Equations (ODE) with Euler and RungeKutta Methods", America Journal of Computational Mathematics,

[7] Kadalbajoo, M. K. "Geometric mesh FDM for selfadjoint singular perturbation boundary value problems", Applied Mathematics and Computation,

[8] Le Veque, R. J. (2007). Finite difference methods for ordinary and partial differential equations: steady-state and timedependent problems. Society for Industrial and Applied Mathematics.

[9] Kaw, A. (2009). Runge-Kutta 4th Order Method for Ordinary Differential Equations. Ordinary Differential Equations, 0804 .

[10] Ralston, A. (1962). Runge-Kutta methods with minimum error bounds. Mathematics of computation, 16 (80), 431-437.

[11] Hull, T. E., Enright, W. H., Fellen, B. M., \& Sedgwick, A. E. (1972). Comparing numerical methods for ordinary differential equations. SIAM Journal on Numerical Analysis, 9 (4), 603-637.

[12] Mungkasi, S., \& Christian, A. (2017, January). Runge-Kutta and rational block methods for solving initial value problems. In Journal of Physics: Conference Series (Vol. 795, No. 1, p. 012040). IOP Publishing.

[13] Butcher, J. C. (1966). On the convergence of numerical solutions to ordinary differential equations. Mathematics of Computation, 20 (93), 1-10.

[14] Griffiths, D. F., Sweby, P. K., \& Yee, H. C. (1992). On spurious asymptotic numerical solutions of explicit RungeKutta methods. IMA Journal of numerical analysis, 12 (3), 319-338.

[15] Islam, M. A. (2015). Accurate solutions of initial value problems for ordinary differential equations with the fourthorder RungeKutta method. Journal of Mathematics Research, $7(3), 41$.

[16] Griffiths, D. F.; Swaby, P. K.; and Yee, Helen C. (1992), On spurious asymptotic numerical solutions of explicit Runge Kutta methods NASA Publications. 244.

[17] Habtamu Garoma Debela and Masho Jima Kabeto (2017) Numerical solution of fourth-order ordinary differential equations using fifth-order Runge - Kuttamethod. Asian Journal of Science and Technology Vol. 08, Issue, 02, pp. 4332-4339.

[18] Shampine L. F. (1984). Stability of explicit Runge-Kutta methods Camp \& Mark with Appi. 7. Vol. IO. No. 6. pp 419432. 1. 